\title{
DESENVOLVIMENTO DO PENSAMENTO CRÍTICO NOS ESTUDANTES DE ENFERMAGEM
}

\author{
DEVELOPMENT OF CRITICAL THINKING \\ IN NURSING STUDENTS
}

\section{DESARROLLO DEL PENSAMIENTO CRÍTICO EN LOS ESTUDIANTES DE ENFERMERÍA}

\author{
Maria do Céu Mendes Pinto Marques \\ Manuel José Lopes ${ }^{2}$ \\ Maria Dulce Domingues Cabral Magalhães ${ }^{3}$ \\ Luís Manuel Mota Sousa ${ }^{4}$
}

Como citar este artigo: Marques MCMP, Lopes MJ, Magalhães MDDC, Sousa LMM. Desenvolvimento do pensamento crítico nos estudantes de enfermagem. Rev baiana enferm. 2022;36:e42849.

Objetivo: analisar o pensamento crítico desenvolvido pelos estudantes participantes de um componente curricular
do curso de graduação em enfermagem. Método: estudo de natureza qualitativa, observacional e comparativa, antes
e após a intervenção. Foi aplicado questionário sociodemográfico e texto-estímulo em dois momentos. A amostra
foi constituída por 48 estudantes que frequentaram a unidade curricular de Pensamento Crítico em Enfermagem.
Resultados: no primeiro momento, os estudantes reconheceram o pensamento crítico como importante na tomada
de decisão e no desenvolvimento de competências, educar para pensar. No segundo momento, os comentários indi-
caram um papel ativo da escola, e a informação e o conhecimento do estudante são acurados no contexto formativo.
Considerações finais: no pensamento crítico desenvolvido pelos estudantes participantes do componente curricular
do curso de enfermagem constatou-se alteração significativa no léxico dos dois momentos, houve desenvolvimento
de habilidades de pensamento crítico, das quais destacamos a interpretação, a argumentação, a análise e avaliação.

Descritores: Pensamento. Conhecimento. Tomada de Decisão Clínica. Educação em Enfermagem. Estudantes de Enfermagem.

Objective: To analyse the critical thinking developed by students participating in a curricular component of undergraduate nursing programmes. Method: Qualitative, observational and comparative study before and after the intervention. A sociodemographic questionnaire and text stimulus were applied in two phases. The sample consisted of 48 students enrolled in the Critical Thinking In Nursing course. Results: In the first phase, the students recognized critical thinking as important in decision-making, in the development of skills and in the process of educating them to think. In the second phase, the comments indicated an active role of the school, and the knowledge of students improved in the formative context. Final considerations: In the critical thinking developed by the students who participated in the curricular component of the nursing programme, a significant change was found in the lexicon in both phases, and critical thinking skills were developed, in particular interpretation, argumentation, analysis, and evaluation.

Keywords: Thought. Knowledge. Clinical Decision-Making. Nursing Education. Nursing students.

\footnotetext{
Professora. Doutora em Psicologia. Professora Coordenadora do Departamento de Enfermagem da Universidade de Évora. Évora, Portugal. mcmarques@uevora.pt. https://orcid.org/0000-0003-2658-3550.

Professor. Doutor em Enfermagem. Professor Coordenador com Agregação do Departamento de Enfermagem da Universidade de Évora. Évora, Portugal. https://orcid.org/0000-0002-7554-804I.

3 Professora. Doutora em Enfermagem. Professora Coordenadora do Departamento de Enfermagem da Universidade de Évora. Évora, Portugal. https://orcid. org/0000-000I-8825-4942.

Professor. Doutor em Enfermagem. Professor Adjunto do Departamento de Enfermagem da Universidade de Évora. Évora, Portugal. https://orcid.org/0000-00029708-5690.
} 
Objetivo: analizar el pensamiento crítico desarrollado por estudiantes que participan en un componente curricular de la carrera de licenciatura en enfermería. Método: estudio cualitativo, observacional y comparativo, antes y después de la intervención. Se aplicó un cuestionario sociodemográfico y un "texto estímulo" en dos momentos. La muestra estuvo constituída por 48 estudiantes que cursaron la unidad curricualar de Pensamiento Crítico en Enfermería. Resultados: en un primer momento, los estudiantes reconocieron el pensamiento crítico como importante en la toma de decisiones y en el desarrollo de habilidades, educar para pensar. En un segundo momento, los comentarios indicaron un papel activo de la escuela, y la información y los conocimientos del alumno son precisos en el contexto formativo. Consideraciones finales: en el pensamiento crítico desarrollado por los estudiantes que participaron en el componente curricular de la carrera de enfermería, bubo un cambio significativo en el léxico de los dos momentos, hubo un desarrollo de habilidades de pensamiento crítico, de las cuales se destacan la interpretación, argumentación, análisis y evaluación.

Descriptores: Pensamiento. Conocimiento. Toma de decisiones clinicas. Educación en enfermería. Estudiantes de enfermería.

\section{Introdução}

O pensamento crítico é referido como um conjunto de habilidades de inferências, suposições, dedução, interpretação e avaliação de argumentos. Também é definido como um julgamento com propósito, de autorregulação, de interpretação, de análise, de avaliação e de inferência ${ }^{(1-2)}$. O pensamento crítico clarifica os objetivos, analisa assunções e evidências, responsabiliza a ação e avalia as conclusões ${ }^{(3)}$. Nesse sentido, é consensual que o pensamento crítico não é um método para ser aprendido, mas sim um processo que exige um conjunto de disposições reconhecidas como características necessárias para pensar criticamente ${ }^{(3-4)}$. Essas disposições impõem a confiança como uma virtude para ser capaz de perspetivar de forma contextualizada, ter criatividade, flexibilidade, curiosidade, ser intelectualmente íntegro, ter capacidade intuitiva, compreensão, firmeza e reflexão; sendo assim, são características necessárias tanto no domínio de um raciocínio cognitivo como afetivo.

O pensamento crítico é definido como o processo mental de perceção, análise, síntese e avaliação ativa e habilidosa da informação recolhida por meio da observação, experiência e comunicação que orienta a construção dos diagnósticos de enfermagem e leva a uma decisão de ação ${ }^{(5-6)}$.

Portanto, pode-se concluir que as competências de pensamento crítico, apesar de se materializarem em decisões, constituem-se essencialmente como um processo mental, uma forma de olhar crítica e criativamente para os dados em presença. Assim, quando se apresenta o pensamento crítico como uma competência e sabendo que estas se manifestam e avaliam na ação, interessa evidenciar que, neste caso, estamos a falar da ação de pensar.

O desenvolvimento dessas competências pode ajudar na problematização e na tomada de decisão e intervenções. Por tais razões, no âmbito da formação em enfermagem, é oferecida como opção uma unidade curricular que é lecionada com recurso a diferentes estratégias que podem capacitar os estudantes para o pensamento crítico, nomeadamente o mapeamento do conceito, a interpretação, a análise e a avaliação de casos clínicos. Estudo refere-se às habilidades ou atributos cognitivos do pensamento crítico como elementos estruturais e essenciais para o desenvolvimento do pensamento crítico, e apresentou a estratégia de ensino baseada na construção de mapas conceituais como eficaz ${ }^{(7)}$. Os mesmos autores, num estudo com estudantes de cursos de graduação em enfermagem, reforçam a ideia da utilização da estratégia de ensino com construção dos mapas conceituais, pois estes apresentam bom desempenho na promoção do Pensamento Crítico Geral e de suas habilidades. Estratégias de ensino baseadas em teorias construtivistas devem ser incentivadas, pois desempenham um papel significativo na melhoria da aprendizagem e do pensamento crítico dos estudantes ${ }^{(8)}$.

Dessa forma, o objetivo deste estudo é analisar o pensamento crítico desenvolvido pelos 
estudantes participantes de um componente curricular do curso de graduação em enfermagem.

\section{Método}

Trata-se de estudo de natureza qualitativa, observacional e comparativa, antes da intervenção e após a intervenção.

O cenário do estudo foi uma Escola Superior de Enfermagem integrada numa universidade do sul de Portugal, no contexto de uma unidade curricular de Pensamento Crítico em Enfermagem, que era oferecida como opção no primeiro e segundo ano curricular do curso de licenciatura em enfermagem. Como estratégia pedagógica recorreu-se a metodologias distintas: métodos verbais individuais com exposição, explicação, diálogo e debates; métodos intuitivos mediante textos escritos, mapeamento de conceitos e trabalhos em grupo com análise crítica e reflexiva sobre estudo de casos.

Participaram do estudo todos os estudantes que frequentaram a unidade curricular de Pensamento Crítico em Enfermagem. Assim, a amostra foi constituída por 48 participantes, maioritariamente do sexo feminino, com média etária de 19 anos, todos estudantes do $1^{\circ}$ e $2^{\circ}$ ano do curso de licenciatura em enfermagem.

A coleta dos dados ocorreu na primeira aula presencial logo após a apresentação formal dos estudantes e do docente, num período de tempo de 30 minutos, e na última aula da unidade curricular. Foi utilizado como instrumento de recolha de dados um questionário sociodemográfico e um depoimento escrito sobre um texto-estímulo, aplicado pelo professor responsável pela unidade curricular. Relativamente aos procedimentos, aplicou-se um texto-estímulo, excerto de "Rebeldes Competentes", retirado de uma palestra do autor ${ }^{(6)}$. Este falou sobre o conceito de "rebeldes competentes" e do facto de não haver "nada melhor" para formar a desobediência que a arte e a criatividade. Segundo o autor "A escola de hoje deve ensinar a pensar, escolher e agir", e sublinhou, ainda, que as escolas devem educar para o fomento do pensamento crítico dos estudantes, formando assim "rebeldes competentes"(6).
Foi solicitada uma análise crítica livre, sem limite do número de palavras, sobre o excerto do texto e também na última aula da unidade curricular, antes dos estudantes terem conhecimento da avaliação final, seguindo as mesmas regras. O propósito de compararmos os resultados encontrados impôs-se, para, dessa forma, percebermos as potenciais alterações no processo de pensamento.

Os dados recolhidos foram sujeitos a análise textual com recurso ao software Interface de $R$ pour les Analyses Multidimensionnelles de Textes et de Questionnaires (IRAMUTEQ) ${ }^{(9)}$. Trata-se de um tipo de análise que permite descrever o material produzido, assim como fazer uma análise relacional. O software realiza análises quantitativas de dados textuais, lexicografia básica até a análise multivariada, por meio da classificação hierárquica descendente (CHD), dando contextos e classes com conteúdos baseados na semelhança dos seus vocabulários. Tem como base a análise, a frequência e a média. Os segmentos de texto são classificados em função do vocabulário, que será repartido conforme a frequência das formas reduzidas.

Com base em matrizes que foram criadas cruzando os segmentos de texto e as palavras em repetidos testes do tipo qui-quadrado, obtém-se uma classificação estável e definitiva. A classe é composta por vários segmentos de texto ou unidades de contexto elementar (uce) segundo a ordenação do vocabulário ${ }^{(9)}$.

Em seguida, os dados foram processados e os segmentos de textos encontrados constituíram os excertos a que recorremos para fundamentar a nomeação das classes. Estes foram analisados e comparados nos dois momentos, recorrendo à análise de conteúdo dos referidos excertos/segmentos de texto.

O estudo teve a aprovação da Comissão de Ética para a Investigação nas Áreas da Saúde Humana e Bem-Estar da Universidade de Évora, Portugal, sob o no 15005 . Todos os participantes foram incluídos no estudo após assinatura do Termo de Consentimento Livre e Esclarecido (TCLE).

Para manter o anonimato dos participantes adotou-se a letra Q (questionário) seguida de número. Essa codificação assegura que a 
identidade do sujeito não será associada às respostas individuais, e os resultados são apresentados de forma que nenhum dos participantes no estudo será reconhecido.

\section{Resultados}

Como referido anteriormente, solicitou-se aos participantes do estudo que, perante o texto-estímulo, produzissem um comentário no início e no final da unidade curricular. Assim, os 48 comentários do $1^{\circ}$ momento constituíram um dos corpus em análise. Na análise textual, a CHD reteve 116 segmentos de texto, correspondendo a $76,32 \%$ do texto total em análise e dividiu o corpus em seis classes, conforme Figura 1.

Figura 1 - Dendograma de classes sobre $1^{\circ}$ momento de comentário ao texto "Rebeldes Competentes"

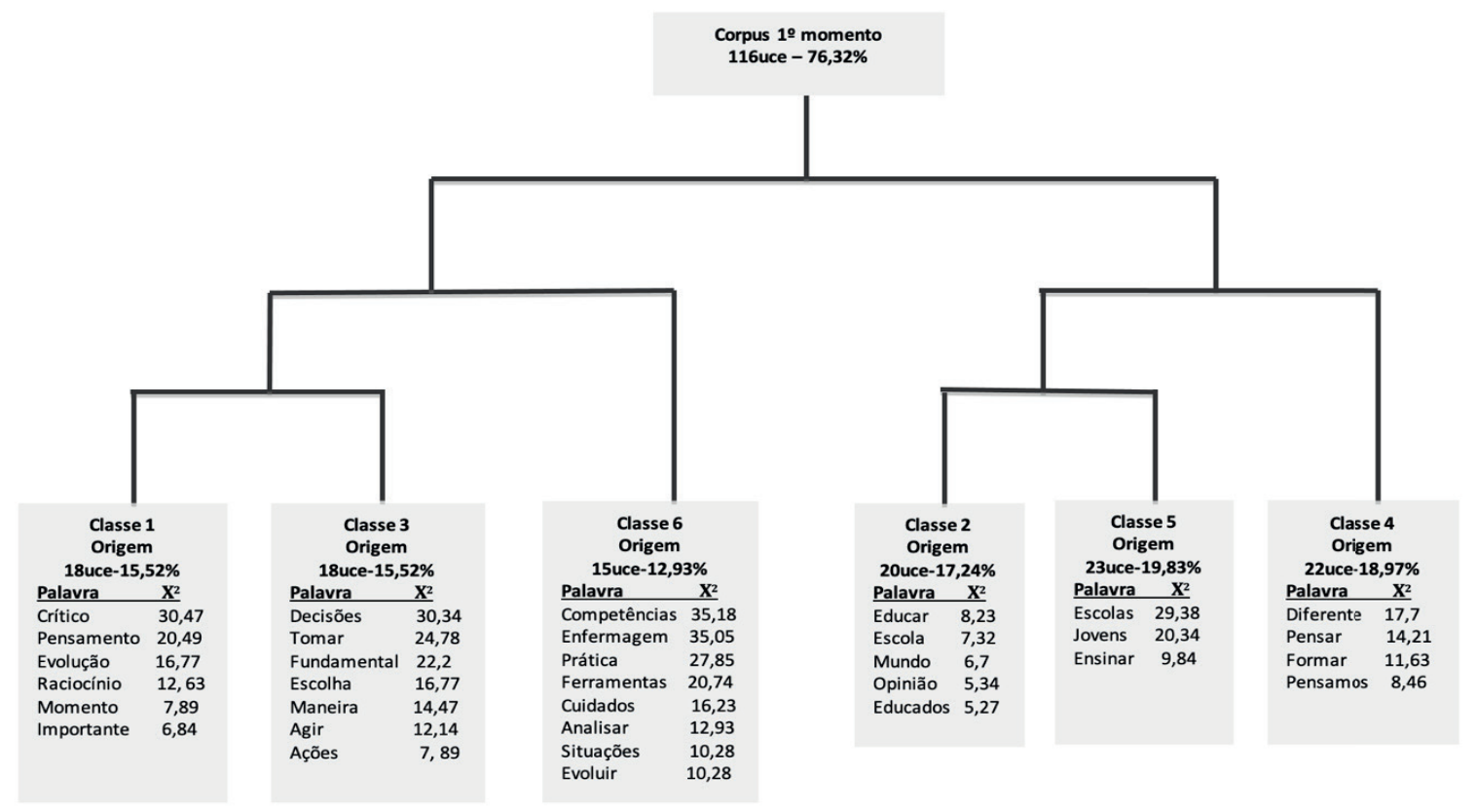

Fonte: Elaboração própria.

Ao analisar o Dendograma da Figura 1, percebe-se claramente uma primeira divisão do corpus em duas partes. A primeira, que engloba as classes 1, 3 e 6, direcionada para a dimensão que releva a importância do "pensamento crítico na tomada de decisão" e, consequentemente, no desenvolvimento de competências, contém menos comentários, 51 uce. A segunda divisão, com as classes 2, 5 e 4, contém uma dimensão marcada pela "escola para educar a pensar", inclui a maioria dos comentários, 65 uce, é, portanto, uma dimensão mais relevante para esses participantes.

Ao analisar as classes de forma individual por ordem de frequência das uces, percebeu-se que, na primeira divisão, na classe 1 , os participantes centram-se no "pensamento crítico como algo importante" e fundamentam da seguinte forma:

[...] o autor defende [...] distinguir o que é certo e o que é errado utilizando o pensamento crítico nas decisões e indecisões da nossa vida [...]. (Q6).

A classe 3 coloca o "pensamento crítico como algo importante lado a lado com a tomada de decisão" e surge fundamentada nos seguintes comentários:

[...] temos assim a capacidade de possuirmos conhecimento para corrigir más práticas [...] colocar questões poder tomar decisões em função do pensamento crítico [...]. (Q4).

Ainda nessa divisão surge a classe 6, que dá especial realce às "competências e sua sustentação", suportada em afirmações a seguir: 
[...] são necessárias ferramentas como o conhecimento para desenvolver competências para uma boa prática clínica [...] a escola uma instituição que deverá apoiar e sustentaro desenvolvimento para o pensamento [...]. (Q8).

$\mathrm{Na}$ segunda divisão do corpus surge a classe 5 com maior percentagem de comentários, focada no "contexto escola" enquanto local de transferência de conhecimento, de entre os quais destacamos:

[...] as escolas entre outras devem educar os alunos para um pensamento crítico [...] devem formar rebeldes competentes no sentido da abrirem horizontes no seu conhecimento [...]. (Q4).

A classe 2 surge ao nível da classe 5 e volta a incidir na escola enquanto entidade que educa para o pensamento (e.g., a escola deve instruir nos alunos uma veia crítica em relação a todos os assuntos da sua vida). A classe 4 revela a necessidade de a escola ser uma entidade formadora de estudantes com "pensamento diferente", centrando-se no estudante, como demonstra o excerto seguinte:

[...] existe crítica e é através dela que conseguimos ultrapassar determinadas atitudes [...]. (Q2).

Os 48 comentários do $2^{\mathrm{o}}$ momento constituíram outro do corpus em análise, referentes ao momento pós-formação. O resultado da análise textual apresenta uma CHD que reteve 146 segmentos de texto, correspondendo a 95,42\% do texto em análise, e dividiu o corpus em sete classes, conforme Figura 2. Esta análise mostra também o corpus dividido em duas partes, que podemos enquadrar em duas grandes temáticas. A primeira, que engloba as classes 7, 4, 3 e 6, tem implícito, por ordem de números de comentários, a "informação" determinada pelo contexto pode "educar para o pensamento coerente" utilizando o conhecimento científico.

Figura 2 - Dendograma de classes sobre o $2^{\circ}$ momento de comentário ao texto "Rebeldes Competentes"

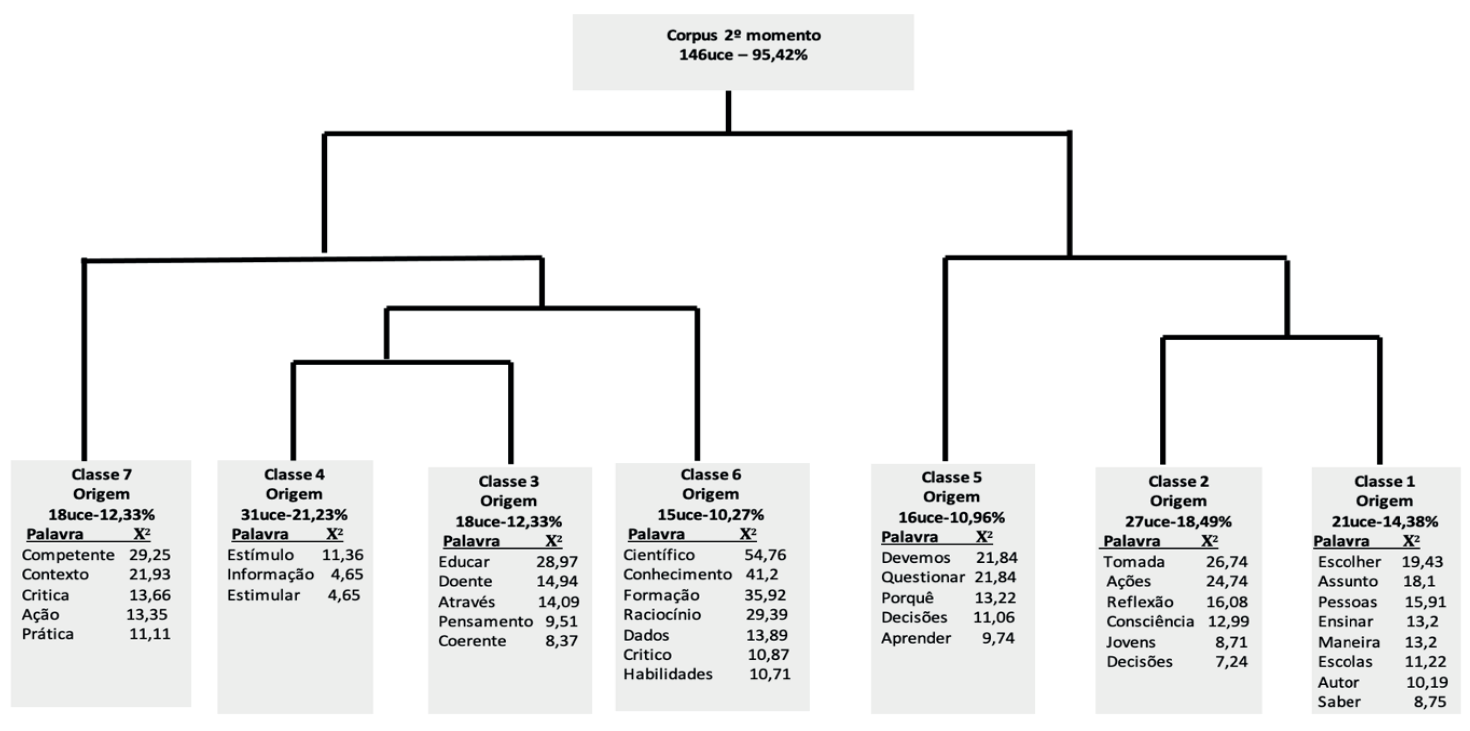

Fonte: Elaboração própria.

Analisando as classes pelo número de comentários, verificou-se que a classe 4 é a de maior percentagem (21,33\%), contudo, com valores de qui-quadrado muito baixos. Essa classe é dominada pelo "estímulo à informação":

[...] quando maior for o estímulo/informação para o pensamento crítico maior será a aquisição de conbecimentos [...]. (Q1).
A classe 7 apresenta duas palavras com elevados valores de qui-quadro, "competente e contexto", que associam à ação e à prática, como clarifica o excerto seguinte:

[...] a abordagem crítica e completa como já foi referido inicia-se com um contexto... avaliar novamente o contexto para reformular a nossa ação [...]. (Q10). 
$\mathrm{Na}$ classe 3 surge o "educar para pensamento coerente", os participantes aludem:

[...] o pensamento crítico deve ser desenvolvido durante o tempo de escola através de exercícios ou situações reais $[\ldots] .(\mathrm{Q} 21)$.

A classe 6 apresenta valores de qui-quadrado muito elevados e demonstra uma tendência de pensamento mais desenvolvido, muito claro nas palavras "conhecimento científico", que pode derivar da "formação" e leva ao "raciocínio". Os participantes já se apropriaram de um conjunto de conceitos que utilizam e consideram importantes, patente em afirmações como:

[...] analisar as situações e delas recolher dados e informações processando essa informação e através do raciocínio clínico da experiência clínica e do conbecimento científico devemos deliberar [...]. (Q7).

[...] pensamento crítico permite avaliar a situação refletir sobre ela levando a tomada de decisão e posteriormente à ação a qual incorpora o conbecimento científico [...]. (Q16).

A segunda divisão envolve, respetivamente, as classes 5, 2 e 1, segundo a ordem de número de comentários. Ao analisar a classe 2, pode-se referir que a "ação" envolve "reflexão" para "aprender a tomar decisões", como refere um dos participantes:

[...] sem esta ferramenta nunca existia uma boa tomada de decisão e mais importante ainda uma reflexão sobre as nossas ações [...]. (Q3).

A classe 1 destaca a dimensão "escola" para "ensinar a escolher", considerada no discurso dos estudantes como pouco explorada, como pode ser observada na asserção seguinte:

[...] o pensamento crítico não é desenvolvido nas escolas, mas deveria ser, pois é através do mesmo que podemos questionar, tentar mudar e saber argumentar [...]. (Q9).

Na classe 5 surge a dimensão "aprender a questionar" para saber o porquê e tomar decisões fundamentadas. Os participantes dão especial valor ao facto de os professores os estimularem a serem elementos ativos na sala de aula, na prática da enfermagem e no desenvolvimento pessoal. Ilustram essa classe da seguinte forma:

[...] estimular os alunos sempre a procurar pelas melhores decisões através da autoconsciência $e$ autorreflexão [...]. (Q2).

\section{Discussão}

Começa-se por salientar que os participantes neste estudo integravam uma unidade curricular opcional denominada Pensamento Crítico em Enfermagem, isto é, os estudantes estavam lá por escolha própria. Considera-se ser um fator determinante no seu desenvolvimento, porque, quando escolheram, já possuíam uma predisposição natural para explorar e desenvolver a temática. A experiência na disciplina proporciona o desenvolvimento do pensamento crítico e reflexivo sobre as ações de enfermagem na perspectiva do cuidado sistematizado e do estabelecimento de vínculos na academia e na clínica com professores e colaboradores do hospital, além de demonstrar a importância da incorporação de evidências científicas para a resolução de problemas da prática clínica, com fins de garantir uma assistência de enfermagem segura e de qualidade ${ }^{(10)}$.

Os resultados encontrados no estudo representacional do processo de desenvolvimento de competências de pensamento crítico dividem-se em dois momentos, antes da intervenção e pós-intervenção. Os dados indicam que os participantes no primeiro momento, antes da intervenção, consideram o desenvolvimento de pensamento crítico como algo necessário na tomada de decisão e, consequentemente, no desenvolvimento de competências. Permite, assim, a representação em construção do ponto de vista conceptual e da aplicabilidade na avaliação, diagnóstico e planeamento das intervenções, já identificada como importante pelos participantes, corroborando o resultado de estudos desenvolvidos por outros autores, que consideram imprescindivel o uso do pensamento crítico no processo de diagnóstico, para alcançar a precisão dos diagnósticos de enfermagem e assistência segura e de qualidade ${ }^{(3,10-12)}$. Ainda em relação aos resultados do primeiro momento do estudo, estes representam uma dimensão marcada pela escola para educar a pensar, resultado que consideramos coerente com os estudos encontrados, no que respeita a responder a questões de 
clarificação, recorrendo à construção de mapas conceituais. Além disso, aprender a pensar e a analisar argumentos desenvolvendo as habilidades ou atributos cognitivos, considerados como elementos estruturais e essenciais para $\mathrm{O}$ desenvolvimento do pensamento crítico ${ }^{(7-8,13)}$.

No segundo momento do estudo, pós-intervenção, observa-se uma evolução muito significativa a nível conceptual e de evolução do conceito de pensamento crítico e sua aplicabilidade enquanto estudantes de enfermagem. Surge o conceito de informação determinado pelo contexto formativo, que deve educar para o pensamento coerente fazendo uso do conhecimento científico. Ideia corroborada por outra pesquisa $^{(11)}$, que se refere ao pensamento crítico como algo que deve ser combinado com o conhecimento e incentivado a pensar sobre o pensamento. Por sua vez, nessa fase pós-intervenção, surge a ação que necessita de reflexão, dando consistência ao processo de aprender a tomar decisões, no qual o contexto escola/universidade tem a função/obrigação de ensinar a aprender e a questionar a realidade com que são confrontados, tanto do ponto de vista teórico como prático. Ideia corroborada por autores ${ }^{(3-4,12)}$, que se referem ao pensamento como auto-dirigido, auto-disciplinado, auto-monitorizado e auto-corretivo, que implica em uma comunicação eficaz na capacidade de resolução de problemas. Essa ideia defendida é de vital importância no desenvolvimento da enfermagem enquanto disciplina, considerada altamente relevante por ter um facilitador teórico e clínico no contexto da prática clínica, visto como um agente que promove reflexão durante a ação. Fundamental para estabelecer aprendizagem cooperativa e uma abordagem colaborativa que promove a integração entre teoria e prática e entre os vários agentes envolvidos, especialmente os estudantes. A avaliação da progressão de competências no contexto da prática clínica do curso de enfermagem permite otimizar estas práticas ao máximo e estabelecer perfis profissionais com maior grau de adaptação para o futuro profissional ${ }^{(14)}$.

O conhecimento científico enunciado pelos estudantes no segundo momento, leva-nos a eleger a prática pedagógica de desenvolvimento dos três elementos da prática baseada em evidências, como uma estratégia de elevado potencial para motivar uma postura mais ativa dos estudantes diante da aquisição de conhecimentos. Ademais, os estudantes tornaram-se conscientes da importância de conhecer as preferências dos pacientes, bem como de valorizar as experiências clínicas construídas durante o cotidiano dos serviços de saúde para a tomada de decisões ${ }^{(15)}$. Esse impulso cognitivo ao desenvolvimento do raciocínio clínico melhora a capacidade diagnóstica dos estudantes, consequentemente, o enunciar de diagnósticos de enfermagem acurados, que favorece a escolha de ações de enfermagem mais adequadas para o alcance dos resultados esperados. Diferentes diagnósticos podem ser enunciados, contudo, o estudante está melhor preparado para priorizar aquele que melhor expressa as respostas humanas do paciente assistido $^{(16)}$. Os achados desta pesquisa apontam que o conhecimento acerca do dinamismo das etapas do Processo de Enfermagem, a aquisição de conhecimento científico, o desenvolvimento do raciocínio clínico e de habilidades intelectuais podem melhorar a capacidade diagnóstica dos futuros enfermeiros e, consequentemente, tendem a aumentar a acurácia dos Diagnósticos de Enfermagem por eles identificados ${ }^{(16)}$.

Unidades curriculares como a do Pensamento Crítico em Enfermagem, à semelhança de outros programas de pensamento crítico, podem estimular o empoderamento dos estudantes de enfermagem e o pensamento crítico-reflexivo de graduandos sobre os aspectos relacionados com o processo saúde-doença e pode atuar como fonte de transformações na formação de profissionais de saúde perante as necessidades de saúde das pessoas ${ }^{(10,16)}$. Contudo, o estímulo à informação que se destaca nas falas dos estudantes pós-intervenção assume especial relevância se for incitado numa dinâmica interdisciplinar, definida como estratégia de ensino de competências, uma vez que começa a ocupar mais espaço na formação de estudantes quando se percebem lacunas na formação do pensamento crítico, com vistas à formação integralizada. A competência 
enquanto estudante, deve fazer parte de toda a formação e ensinada de forma integrada ao longo do currículo, com o objetivo de ensinar a base cognitiva de forma contínua e processual, de modo que o estudante internalize os atributos de competência e sua importância no desempenho de assistência ao paciente; motivo pelo qual esta unidade curricular era lecionada no segundo semestre do primeiro ano do curso ${ }^{(17)}$.

Aprender a questionar tal como referiram os estudantes no segundo momento, leva o professor a ponderar aplicar outras estratégias de ensino-aprendizagem, que podem ser geradoras de satisfação e autoconfiança. A simulação, uma das mais faladas, mas ainda pouco utilizada na sua essência, e as estratégias tradicionais podem ser utilizadas mutuamente na formação em enfermagem. A educação baseada em simulação é um componente importante na preparação dos estudantes visando uma transição bem-sucedida para a prática clínica. Permite que o estudante treine as competências de avaliação diagnóstica, ação e avaliação dos resultados, as vezes necessárias, até obter segurança na tomada de decisão e na ação. Além disso, contribui para a satisfação, conhecimento, confiança, competência e pensamento crítico do estudante ${ }^{(18-19)}$.

A formação dos futuros enfermeiros deve ser construída na égide da relação educador-estudantes e demais participantes no momento da aprendizagem, mediante um diálogo inclusivo dos fatos reais associados ao raciocínio clínico e à prestação de cuidados de qualidade. Essa comunicação tem de ser mediada por discurso coerente, receptivo e reflexivo num contexto situacional entre os sujeitos, de modo a contribuir para a formação profissional dos enfermeiros, com especial enfoque no desenvolvimento de competências de pensamento crítico em Enfermagem ${ }^{(14-16,20)}$.

Este estudo poderá contribuir na prática do ensino de enfermagem, uma vez que deu contributo para a compreensão do desenvolvimento do pensamento crítico e consequente tomada de decisão na avaliação, diagnóstico e planeamento das ações de enfermagem, fundamental para a precisão dos diagnósticos de enfermagem.
Além disso, acredita-se que a compreensão do desenvolvimento do pensamento crítico em enfermagem poderá contribuir para futuras intervenções e estudos que avaliem o desenvolvimento desse processo, servindo como base para a elaboração e implementação de estratégias de ensino que tenham como objetivo estimular as competências de pensamento crítico em todo o processo de enfermagem.

A principal limitação deste estudo está relacionada com o fato de ter sido realizado somente com estudantes de uma escola de enfermagem de uma universidade pública, pelo que se recomenda, em estudos futuros, a inclusão de outras instituições de ensino e escolas de enfermagem públicas e privadas.

\section{Considerações Finais}

Ao analisar o pensamento crítico desenvolvido pelos estudantes participantes numa unidade curricular, constatam-se semelhanças e diferenças entre o primeiro e o segundo momento em análise.

De entre as semelhanças, destacamos a importância atribuída ao pensamento crítico. Compreende-se que, sendo esta uma unidade curricular opcional, significa que os estudantes que a escolhem têm alguma apetência prévia para desenvolver esta formação.

De entre as semelhanças, destaque ainda para o papel atribuído à Escola no processo de formação para o pensamento crítico. Esta é assim entendida como promotora do inconformismo subjacente a um pensamento que se quer "rebelde" criativo e crítico.

Relativamente às diferenças, assinala-se essencialmente as que têm relação com uma acrescida capacidade de análise e de inferência sobre e a partir do texto e uma alteração significativa no léxico utilizado nos dois momentos, o que denota um desenvolvimento de habilidades de pensamento crítico, das quais destacamos a interpretação, a análise e a avaliação.

Pode-se, assim, afirmar que a metodologia utilizada em sala de aula permitiu aos estudantes manifestar disposições necessárias para 
o pensamento crítico. O facto de serem usadas diferentes estratégias permitiu que os estudantes mergulhassem numa apreensão e compreensão do conceito de pensamento crítico. Isso é reconhecido pelo uso sistemático de indicadores que são consensuais entre os autores e que denunciam as disposições necessárias para o pensamento crítico.

Os resultados do estudo permitem afirmar que a escola pode educar para uma rebeldia competente. Esse processo de apropriação de habilidades em pensamento crítico em estudantes deve ser continuado. Ele não é estático, requer um acompanhamento e incitamento constante interdisciplinarmente, assim como didáticas apropriadas à sua efetivação. Considera-se que o pensamento crítico é essencial ao desenvolvimento dos estudantes de enfermagem, pois estimula as suas capacidades de criatividade, flexibilidade, curiosidade, integridade intelectual, capacidade intuitiva, compreensão, perseverança e reflexão nas atividades do quotidiano e na prática de enfermagem.

\section{Colaborações:}

1 - concepção, projeto, análise e interpretação dos dados: Maria do Céu Mendes Pinto Marques e Manuel José Lopes;

2 - redação do artigo e revisão crítica relevante do conteúdo intelectual: Maria do Céu Mendes Pinto Marques, Manuel José Lopes e Dulce Magalhães;

3 - aprovação final da versão a ser publicada: Maria do Céu Mendes Pinto e Luís Sousa.

\section{Referências}

1. Watson G, Glaser EM. Watson-Glaser critical thinking appraisal. New York: The Psychological Corporation; 1980.

2. Facione PA. Critical Thinking: A Statement of Expert Consensus for Purposes of Educational Assessment and Instruction. "The Delphi Report" [Internet]. Millbrae (CA): California Academic Press; 1990 [cited 2019 Sep 20]. Available from: https:// www.qcc.cuny.edu/socialsciences/ppecorino/CTExpert-Report.pdf
3. Alfaro-LeFevre R. Critical Thinking and Clinical Judgment: A practical approach. St Louis (MO): Elsevier Saunders; 2013.

4. Facione PA. Critical Thinking: What It Is and Why It Counts. Insight Assessment [Internet]. 2011 Jun [cited 2019 Oct 10];2007(1):1-23. Available from: https://www.insightassessment. com/CT-Resources/Teaching-For-and-AboutCritical-Thinking/Critical-Thinking-What-It-Isand-Why-It-Counts/Critical-Thinking-What-It-Isand-Why-It-Counts-PDF

5. Dominguez C, coordenadora. Pensamento crítico na educação: desafios atuais [Internet]. Vila Real: UTAD; 2015 [cited 2019 Sep 30]. Available from: https://www.pensamiento-critico.com/archivos/ ebookutad.pdf

6. Lúcio L. Escola deve estimular pensamento crítico dos alunos diz ex-ministro da Justiça [Internet]. Lisboa (PT): 2009 [cited 2019 Oct 10]. Available from: https://www.publico.pt/2009/03/10/portugal/ noticia/escola-deve-estimular-pensamento-criticodos-alunos-diz-exministro-da-justica-1368618

7. Carvalho DPSRP, Vitor AF, Cogo ALP, Bittencourt GKGD, Santos VEP, Ferreira Júnior MA. Pensamento crítico em estudantes de Enfermagem de duas regiões brasileiras. Rev Bras Enferm. 2020;73(1):e20170742. DOI: https://doi.org/10.1590/ 0034-7167-2017-0742

8. Carvalho DPSRP, Vitor AF, Cogo ALP, Bittencourt GKGD, Santos VEP, Ferreira Júnior MA. Mensuração do pensamento crítico geral em estudantes de cursos de graduação em enfermagem: estudo experimental. Texto contexto - enferm. 2020;29:e20180229. DOI: https://doi.org/10.1590/1980-265x-tce-2018-0229

9. Camargo VB, Justo AM. Tutorial para uso de software de análise textual IRAMUTEQ [Internet]. Florianópolis; 2013. [cited 2019 Oct 8]. Available from: http://www.iramuteq.org/documentation/ fichiers/tutoriel-en-portugais

10. Lopes JM, Pereira AG, Pereira LCS, Magnabosco P, Figueiredo VN, Ferreira MBG. Vivência prática de acadêmicos de enfermagem na unidade de clínica médica: relato de experiência. Braz J Health Rev. 2020 May 1;3(3):4351-6. Available from: http://www.brazilianjournals.com/index. php/BJHR/article/view/9867/8292

11. Lunney M. Use of Critical Thinking in the Diagnostic Process. Int $\mathrm{J}$ Nurs Terminol 
Classif. 2010;21(2):82-8. DOI: https://doi.org/ 10.1111/j.1744-618X.2010.01150.x

12. Benner P, Hughes RG, Sutphen M. Clinical Reasoning, Decisionmaking, and Action: Thinking Critically and Clinically. In: Hughes RG, editor. Patient Safety and Quality: An Evidence-Based Handbook for Nurses. Rockville (US): Agency for Healthcare Research and Quality; 2008. p. 87-110. Available from: https://www.ncbi.nlm.nih.gov/ books/NBK2643/

13. Tenreiro-Vieira C, Vieira RM. Literacia e pensamento crítico: um referencial para a educação em ciências e em matemática. Rev Bras Educ. 2013;18(52):163-88. Available from: http://w. scielo.br/pdf/rbedu/v18n52/10.pdf

14. Martínez-Momblán MA, Colina-Torralva J, Cueva-Ariza LD, Guix-Comellas EM, RomeroGarcía M, Delgado-Hito P. Análise da evolução de competências da prática clínica no curso de enfermagem. Rev Latino-Am Enferm. 2020;28:e3231. DOI: http://dx.doi.org/ $10.1590 / 1518-8345.2927 .3231$

15. Ferraz L, Schneider LR, Pereira RP, Pereira AM. Ensino e aprendizagem da prática baseada em evidências nos cursos de Enfermagem e Medicina. Rev Bras Estud Pedagógicos. 2020;101(257). DOI: http://dx.doi.org/10.24109/ 2176-6681.rbep.101i257.4424

16. Silva AM, Bertoncello KCG, Silva TG, Amante LN, Matos FGOA, Bellaguarda MLR. Acurácia de diagnósticos de enfermagem: revisão integrativa. Rev Enferm Brasil. 2020;19(2):167-75. DOI: https:// doi.org/10.33233/eb.v19i2.3078

17. Costa LS, Formozo GA. Representações sociais de graduandos acerca do programa educação pelo trabalho para saúde. Rev Bras Enferm. 2018;71(2):244-51. DOI: http://dx.doi.org/10.1590/ 0034-7167-2015-0168

18. Marçal ARV, Zagonel IPS. Profissionalismo na formação de enfermeiros: apreensão das significações de docentes e estudantes. J Nurs Health. 2020;10(1):e20101008. DOI: http://dx.doi. org/10.15210/jonah.v10i1.18160

19. Costa RRO, Medeiros SM, Coutinho VRD, Mazzo A, Araújo MS. Satisfação e autoconfiança na aprendizagem de estudantes de enfermagem: ensaio clínico randomizado. Esc Anna Nery. 2020;24(1):e20190094. DOI: http://dx.doi.org/10.1590/ 2177-9465-ean-2019-0094

20. Carvalho DPSRP, Vitor AF, Cogo ALP, Santos VEP, Ferreira Júnior MA. Teoria da ação comunicativa: subsídio para o desenvolvimento do pensamento crítico. Rev Bras Enferm. 2017;70(6):1414-7. DOI: http://dx.doi.org/10.1590/ 0034-7167-2016-0383

Recebido: 21 de dezembro de 2020

Aprovado: 21 de outubro de 2021

Publicado: 16 de fevereiro de 2022

A Revista Baiana de Enfermagem utiliza a Licença Creative Commons - Atribuição-NãoComercial 4.0 Internacional.

https://creativecommons.org/licenses/by-nc/4.0/

Este artigo é de acesso aberto distribuído sob os termos da Licença Creative Commons (CC BY-NC).

Esta licença permite que outros remixem, adaptem e criem a partir do seu trabalho para fins não comerciais. Embora os novos trabalhos tenham de lhe atribuir o devido crédito e não possam ser usados para fins comerciais, os usuários não têm de licenciar esses trabalhos derivados sob os mesmos termos. 